\title{
3D Visualization of Motor-Neurons in Mice Spinal Cord Using FIB/SEM Tomography
}

\author{
Binbin Deng ${ }^{1}$, Camila M. Freria ${ }^{2}$, Robert E.A. Williams ${ }^{1}$, Daniel Huber ${ }^{1}$, John Sosa ${ }^{1}$, Philip G. \\ Popovich $^{2}$, David W. McComb ${ }^{1}$ \\ ${ }^{1}$ Department of Materials Science and Engineering, The Ohio State University, Columbus, OH 43210 \\ USA \\ 2 Department of Neuroscience, The Ohio State University, Columbus, OH 43210 USA
}

\begin{abstract}
After spinal cord injury (SCI) axonal pathways are disrupted at the lesion, which results in loss of motor, sensory and autonomic function. To re-establish synaptic circuits, survived neurons extend their axons to develop functional synapses. Inflammation is a localized defensive response of tissue to lesion and is essential for neural repair. Microglia and macrophages are activated and recruited to the lesion. Research suggested that before and after SCI glia cells regulate many of the signaling mechanisms that control the neural repair ability [1-4]. Several studies also showed direct interactions between microglia and neurons play a crucial role in health and disease. Dual-beam focused ion beam (FIB) instruments are extensively used in materials science, and are increasingly being used to investigate three dimensional (3D) structures of biological materials [5]. In this contribution we report the use of FIB/SEM tomography in the structural study of motor-neurons in the lumbar spinal cord of mice after SCI.
\end{abstract}

Mice spinal cord tissue was fixed in $0.15 \mathrm{M}$ cacodylate buffer containing $2.5 \%$ glutaraldehyde, $2 \%$ formaldehyde. Fixed tissue was cut into 200-500um thick sections. Tissue sections were immersed in a solution with $3 \%$ potassium ferrocyanide in $0.3 \mathrm{M}$ cacodylate buffer with $4 \mathrm{mM}$ calcium chloride that was combined with an equal volume of $4 \%$ aqueous osmium tetroxide for 1 hour at room temperature. After rinse, tissue was immersed in filtered 1\% Thiocarbohydrazide for 20 minutes, and then immersed in $2 \%$ osmium for 30 minutes. Rinsed tissue was then left overnight in $1 \%$ uranyl acetate at $4{ }^{\circ} \mathrm{C}$. Rinse the tissue and immerse it in lead aspartate solution for $30 \mathrm{~min}(0.066 \mathrm{~g}$ lead nitrate dissolved in $10 \mathrm{ml}$ of $0.003 \mathrm{M}$ aspartic acid solution, $\mathrm{pH}$ adjusted to 5.5 with $1 \mathrm{~N} \mathrm{KOH}$, warmed in a $60^{\circ} \mathrm{C}$ ). Rinse and dehydrate tissue in graded alcohols $(30 \%, 50 \%, 70 \%, 80 \%, 90 \%, 2 \times 100 \%)$ for 15 minutes each. Immerse tissue in 1:0, 3:1, 1:1 and 1:3 acetone/resin for 30 minutes each. Leave the tissue in $100 \%$ resin overnight. After incubate at $65^{\circ} \mathrm{C}$ for 2 days, the resin embedded tissue was cut and processed by microtome and then mounted on a SEM stub. Electron micrographs were collection on Helios Nanolab 600 DualBeam (FIB/SEM) (FEI, Hillsboro). Images were processed using MIPAR software and visualized using Aviso.

Neurons and microglia were clearly identified in 3D volume. The result demonstrated that FIB/SEM is a suitable investigation method for structural study in spinal cord injury. Currently there are no effective pharmacological standard of care therapies available to enhance neural repair and recovery. 3D visualization of spinal cord will provide unprecedented insights into the interactions between microglia and neuron after SCI which may serve as potential therapeutic targets. 


\section{References:}

[1] NJ Allen et al. "Signaling between glia and neurons: focus on synaptic plasticity", Curr Opin Neurobiol. 2005; 15(5): p542-548

[2] FW Pfrieger, "Roles of glial cells in synapse development", Cell Mol Life Sci. 2009; 66(13): p2037-2047

[3] C Eroglu et al. "Regulation of synaptic connectivity by glia", Nature. 2010; 468 (7321): p223-231

[4] H Kettenmann et al. "Microglia: new roles for the synaptic stripper", Neuron. 2013; 77(1): p10-18

[5] Bushby et al. "Imaging three-dimensional tissue architectures by focus ion beam scanning electron microscopy", Nature protocols 2011; 6(6): p845-858

[6] The authors acknowledge funding from The Ohio Third Frontier Program and the Center for Regenerative Medicine and Cell-based Therapies and The Center for Electron Microscopy and Analysis at The Ohio State University

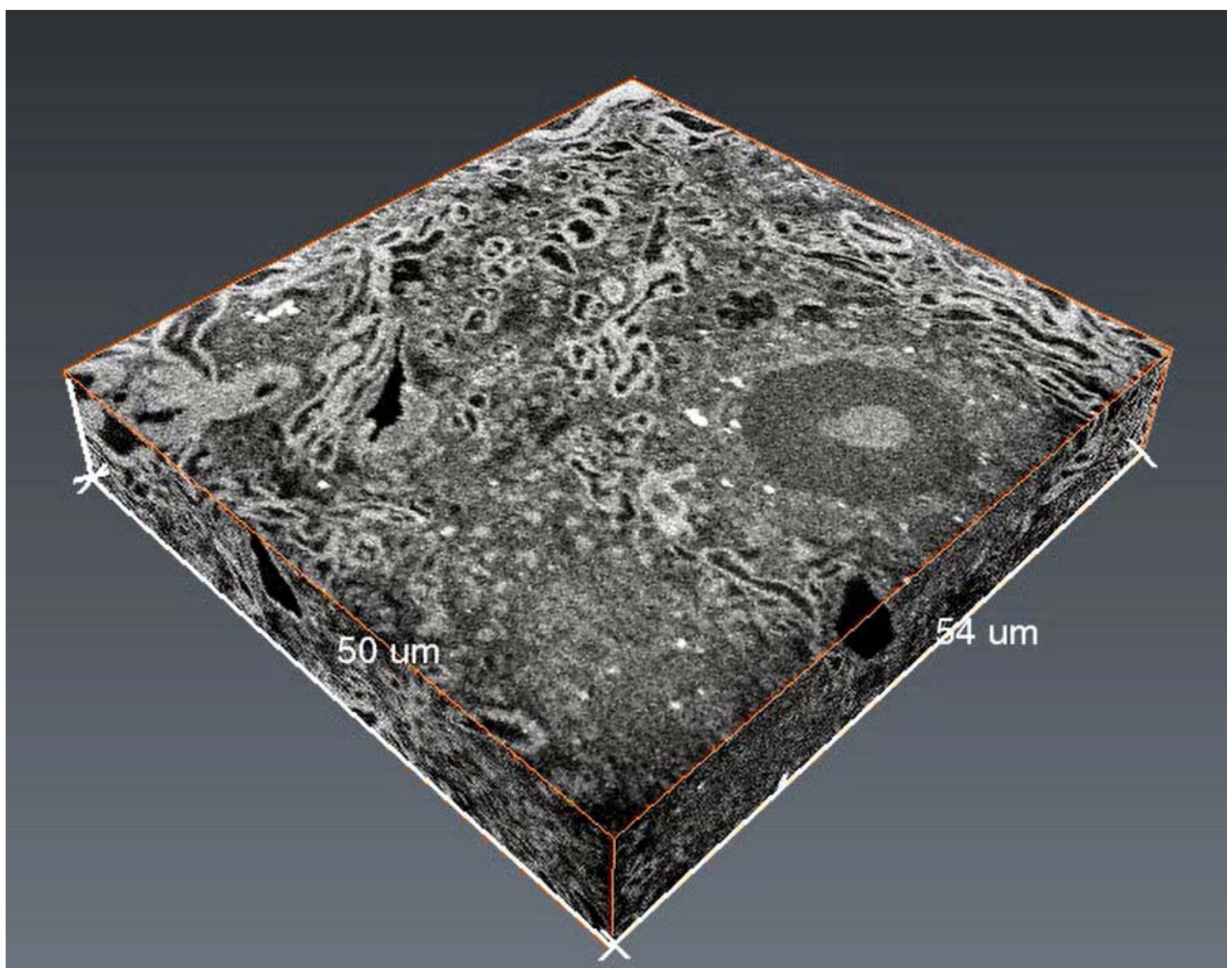

Figure 1. 3D volume view of mice spinal cord tissue obtained using FIB/SEM tomography 